\title{
EVALUATION OF AN ACTIVE SAFETY LIGHT USING VIRTUAL TEST DRIVE WITHIN VEHICLE IN THE LOOP
}

\author{
*Yvonne Laschinsky, **Kilian von Neumann-Cosel, *Dr. Mark Gonter, *Christian Wegwerth, \\ ****Dr. Rolf Dubitzky, **Prof. A. Knoll \\ * Volkswagen AG \\ Group Research \\ Letter box 1777, 38436 Wolfsburg \\ ** TUM - Technische Universität München \\ Informatik VI: Robotik \& Embedded Systems \\ Boltzmannstr. 3, D-85748 Garching \\ *** Carmeq GmbH \\ Carnotstraße 4 \\ D-10587 Berlin
}

yvonne.laschinsky@volkswagen.de,neumannc@in.tum.de,mark.gonter@volkswagen.de, christian.wegwerth@volkswagen.de,rolf.dubitzky@carmeq.com, knoll@in.tum.de

\begin{abstract}
Driving at night time increases the risk of accidents because the driver's perception is reduced to the limited area of the environment which is illuminated by the car's head lights. In the study presented in this paper, the tool Vehicle in the Loop (VIL) is used the first time to research the potential of a new light function. VIL is a Virtual Reality application and simulation setup which allows for testing driver assistance systems in critical driving situations while driving a real car, but without the risk of collisions with real objects. VIL is based on the Virtual Test Drive (VTD) simulation software. The Active Safety Light is an advanced light functionality which illuminates the potential escape path a driver should take in order to avoid an imminent accident. The two main results of this first field study are, that VIL is suitable to study advanced light functions and that the Active Safety Light supports the driver in critical situations. The driver reacts nearly $60 \mathrm{~ms}$ faster and the situation is estimated as more critical so that the driver brakes instinctively harder, i.e. the break pressure is increased by almost a factor of two.
\end{abstract}

\section{INTRODUCTION}

Darkness increases the risk of accidents for drivers and pedestrians. Less light intensity, lower contrasts and reduced visibility are just a few reasons why driving at night causes difficulties. Therefore it is important to illuminate the environment as well as possible to increase security by a good light performance [1].
The development, test, and presentation of driver assistance systems pose increasingly challenging difficulties for the developers. In the past, there have been only two options for research and for testing new functions in this rapidly growing segment of automotive electronics: Driver assistant systems that support the driver in critical traffic situations are tested either in real experiments with foam cubes and other kind of mockups, like specially protected target vehicles, or they have been tested in driving simulators.

On one hand, the traffic situations in which the assistance system is supporting the driver are becoming more complex and, thus are more difficult to reproduce in setups with real vehicles and targets. On the other hand, the emerging concepts of haptic and kinesthetic human machine interfaces make the use of motionless simulators almost impossible, or require a high level of realism from motion simulators. State of the art motion driving simulators are still, at best, imperfect, which leads to results which are hard to transfer into reality and to many other problems like motion sickness.

The test- and simulation environment Vehicle in the Loop, developed by Audi AG opens up a huge space of new opportunities by combining the best of two worlds, i.e. the safety, reproducibility and flexibility of driving simulators and the perfect motion dynamic of a real vehicle. The aim of the 
current study is the validation of light functions and the examination of their effect potential.

\section{DESCRIPTION OF THE LIGHT FUNCTION}

On basis of the accident analysis of the target population using the GIDAS-Database, one can see that many night-time accidents could have been avoided by an active light application. The "Adaptive Cut-Off Line" and the "Masked High Beam" are the first step for improving visibility at night. The driver sees more and reacts earlier in dangerous situations. Further challenges are scenarios where an object suddenly appears or where the driver is looking in another direction because of disturbing light sources.

These are scenarios where the Active Safety Light has the potential to help the driver. For example, a pedestrian who appears unexpectedly walking on the road can be recognized much earlier by the driver with a Marking Light. The Marking Light guides the viewing to the direction of the pedestrian. Objects will be illuminated by light spots after they are analyzed by environmental sensors [1].

\section{VIRTUAL TEST DRIVE SIMULATION ENVIRONMENT}

The simulation software running within the Vehicle in the Loop is called Virtual Test Drive (VTD) [2]. VTD consists of several types of components: core components that handle the data flow within the simulation, extended components that are used to simulate the vehicle and environment, and target components which will actually be contributed by the developers of an assistance system.

One of the crucial software components to simulate an active light system is the visualization that graphically renders the virtual scene. The Image-Generator (IG), one of Virtual Test Drive's extended components, allows the user to set up a night environment including cars with headlights illuminating their surroundings.

It is possible to set-up different illuminating profiles for each headlight. One Profile is defined by a two-dimensional greyscale image that shows an illumination map. This map is the 2D luminosity distribution in a plane perpendicular to the $\mathrm{x}$ coordinate axis in the car's base coordinate system (DIN70000), i.e. perpendicular to the car's direction of driving. The profiles can be changed during the simulation to cover the Active Safety Light functionality. The frustum and intensity of the light beam is configurable, as well as, the reflective properties of the environmental mapping functions for ambient, diffuse, and specular components.

At runtime, the position $(\mathrm{x}, \mathrm{y}, \mathrm{z})$ and orientation (heading, pitch, roll) of the lights can be changed in real time via an input interface to VTD. The position can either be given in absolute coordinates for fixed lights, or in coordinates relative to other cars or the ego-vehicle for moving lights like head lamps. The same interface is used to switch between the preconfigured illumination maps. In this study, three different illuminations

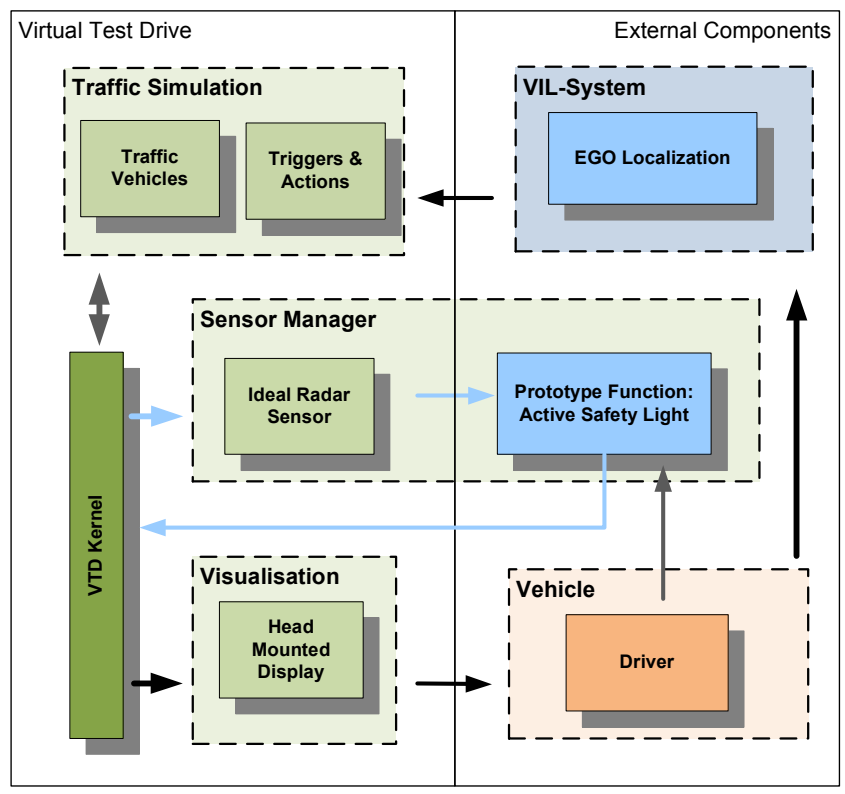

Fig. 1. Testbed Architecture

have been used: low beams, high beams, and the active safety light.

The Active Safety Light assistance function is connected to the VTD core component via the output interface of VTD. The interface communicates the positions of all traffic objects, such as e.g. vehicles, to the sensor manager component, as shown in figure 1. The Sensor Manager contains a sensor model plug-in which simulates an ideal radar sensor, i.e. the sensor has a large horizontal opening angle and perfect tracking and detection efficiency. It is possible to simulate more realistic sensor models in VTD, but for this study it is important that the function being tested can rely on perfect sensor data.

Based on this information, a prototypical function is calculating the time to collision and activating the safety light approximately 1-2 seconds before a potential crash. Since coordinates of virtual opponent vehicle are known from the sensor model output, it is possible to calculate the right orientation of the active safety lights and to send the corresponding coordinates to the IG component. The resulting image is shown in figure 2.

\section{VEHICLE-IN-THE-LOOP SIMULATOR}

The visual output of the IG is connected to a head mounted display that the driver of the Vehicle in the Loop car wears. Since the display has optical see-through capabilities, the virtual reality can span from mere vehicles which are projected on a real street (augmented reality) up to a full featured virtual reality where the driver has no perception of the real world. In this mode, driving is only possible in large, open spaces which can be found in test areas or air fields.

Since using Vehicle in the Loop means driving a real vehicle in the real world of a test facility, there is no need to simulate 
vehicle dynamics [3]. The real vehicle dynamics are measured in six degrees of freedom with an inertial measurement unit, acceleration sensors, gyroscopes and differential GPS (DGPS). This measurement is fed in real time into the VTD simulation core.

Since the visual component of the system is a head mounted display, it is also necessary to measure the position of the driver's head in six degrees of freedom. This is done using a laser head tracker (laserBIRDII, Ascension Tech). Its data is also fed into the simulation core. After a calibration procedure, which determines relative positions of all detectors with respect to each other and to the vehicle itself, it is effectively possible to measure the position of the drivers head in the virtual environment and thus, to generate the appropriate 3D computer graphics for the drivers view.

VIL is compact and mobile (Fig. 3). It can be built into cars of any make and type with little effort. It connects to the vehicle's onboard power and data networks, be it CAN, FlexRay, or IP based communication.

The connection to the vehicle's onboard communication system is important for two reasons. On the one hand, it allows to record events in the real world like the vehicle's state and the driver's actions, steering, braking, accelerating time synchronous with events in the virtual reality, like positions and actions of virtual vehicles and pedestrians. This allows an easy and effective analysis of recorded data. On the other hand, it enables the VIL-system to access the vehicle's capabilities from optical, acoustic, kinesthetic warning channels up to full control of the vehicle's power train and steering. The options are only limited by the test carrier's features.

The realism of VIL can be shown by the fact that the numbers of test subjects which suffer from motion sickness are

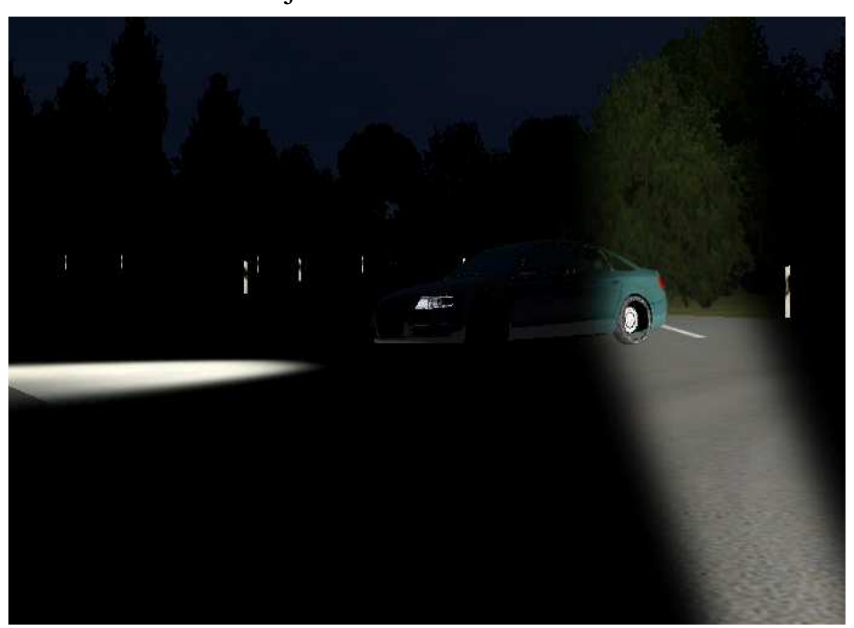

Fig. 2. Illumination of the opponents back

less than $10 \%$ when driving in a full virtual reality setup. When used in augmented reality setups there has not been a single case of motion sickness in tests on more than 100 people [6].
For this study, the system was mounted into a Volkswagen Passat CC test carrier. The system was connected to the vehicles drive train CAN-bus. This allows the recording of all parameters necessary to analyze the test person's reaction to the Active Safety Light function, such as angle of the steering wheel, position of gas and brake pedal, break pressure and several others. Since the position of the light switch is mirrored via a CAN-gateway to the same CAN-bus, information about the status of the vehicle's lights is available to the VIL-system.

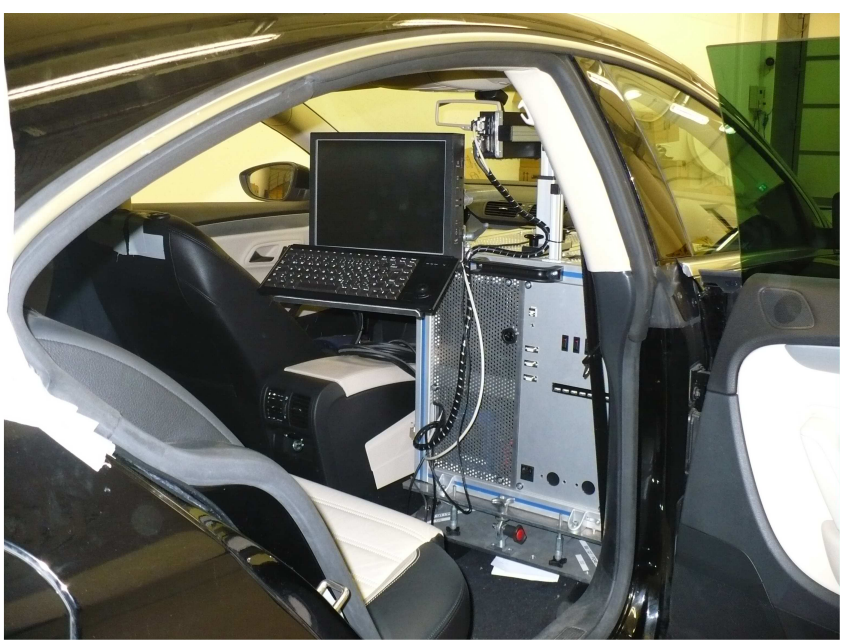

Fig. 3. VIL simulator in motion

Depending on the status of the real car's lights, the function switches the virtual vehicle's head lights from off to low beams, or to high beams. This interaction between real world (light switch) and virtual world (low, high beam illumination) further improves the realism of the setup.

The Active Safety Light illuminates the trunk of the opponent car (Fig. 2). The light is constantly adjusted while the opponent car is crossing the road in front of the ego-vehicle.

\section{DESCRIPTION OF THE SCENARIO}

In the German in depth database (GIDAS) one can see that accident partners react incorrectly in many critical situations. They steer in the same direction and collide. An Active Safety Light could assist the action of a driver so that he intuitively steers in the right direction. [1]

The entire scene is based on a rural environment. In order to represent the scenario as realistically as possible, other vehicles and humans are also shown. At the beginning, the driver crosses four junctions and no critical situation occurs. At the next junction, another vehicle suddenly approaches from the right, and then turns left, taking the driver's right of way. However, if the driver were to continue with constant speed, the vehicle coming from the right would be fast enough to avoid a collision. 

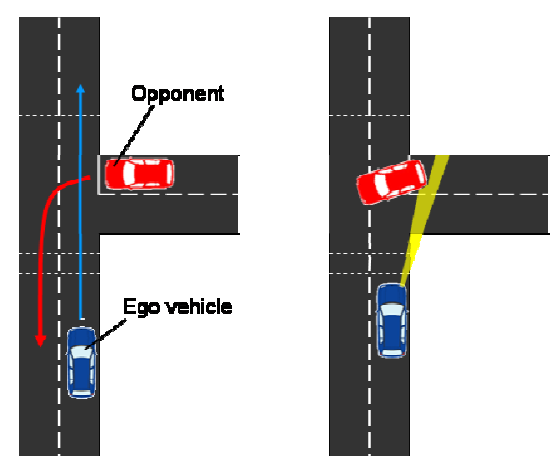

Fig. 4. ASL Scenario

After the target car crosses the driver's vehicle's trajectory, the Active Safety Light simulation is enabled. It shrinks the headlights of the driver's car so that it only illuminates its own lane and shines strongly at the tail of the target car. This should prevent the driver from being irritated by the target car's headlight and accidentally colliding with him.

\section{RESULTS}

The Objectives of the test study were the evaluation of the Active Safety Light function, as well as, the suitability of the Vehicle-in-the-Loop (VIL) for light function simulation. The test study showed that the VIL has predominant advantages. In the following the experiences with the VIL are listed in positive $(+)$ and negative (-) properties.

$+\quad$ Realistic illustration of the real world

$+\quad$ Fast adaptation on the visual world

$+\quad$ Driving behavior in critical situations like in reality

$+\quad$ The hardware can be installed in every vehicle

$+\quad$ VIL can be used for light function tests at daytime

- $\quad$ The helmet is uncomfortable

- The viewing angle is limited

- Some of the test persons felt dizzy after the experiments

The results of the Active Safety Light function are different from the experiences of the GIDAS database. None of the drivers without active light function steered in the direction of the opponent car. However, some effects could be seen which are very interesting for future Active Safety Light applications. With the Active Safety Light function, the driver reacts nearly $60 \mathrm{~ms}$ faster and the situation is estimated as more critical so that the driver brakes instinctively harder. (Fig. and Fig. ) As a result of the hard break, the drivers of the group (VG2) with Active Safety Light function steered less than the other group (VG1). (Fig. )

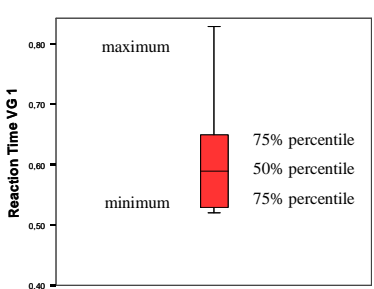

Fig. 5: Reaction Time

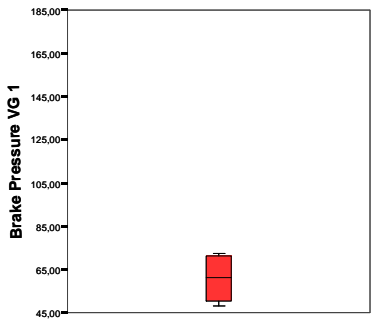

Fig. 6: Brake Pressure

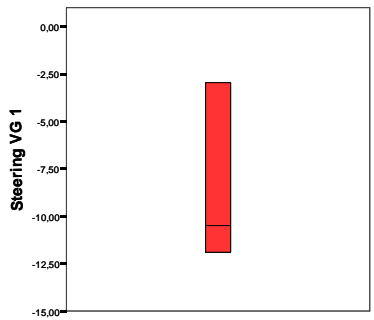

Fig. 7: Steering
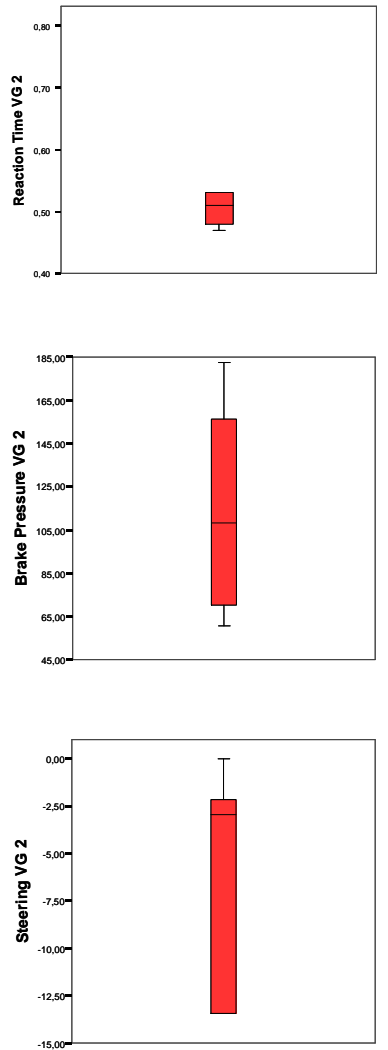

\section{CONCLUSION}

It is apparent that it is possible to influence the reaction of the driver, but it is rather difficult to find the right light application. So, we have to focus our research on controlling the gaze direction, decreasing the reaction time and influencing the driver's action. With the help of the Vehicle-in-the-Loop, we will develop new test scenarios with new light applications to evaluate the potential of Active Safety Light functions

\section{REFERENCES}

[1] C. Wegwerth, M. Gonter, Y. Laschinsky, J. Hilgenstock, S. Thomschke, Active Safety Light, in proceedings on Vision Conference, 2008, Paris, Frankreich.

[2] K. von Neumann-Cosel, M. Dupuis, C. Weiss, Virtual Test Drive Provision of a Consistent Tool-Set for [D,H,S,V]-in-the-Loop, In Proceedings on Driving Simulation Conference Europe, 2009, Monaco, provided that this paper will be accepted by the DSC scientific committee

[3] Th. Bock, M. Maurer, Prof. Georg Färber, Vehicle in the Loop - A new simulator set-up for testing Advanced Driving Assistance Systems; Driving Simulation Conference 2007, Iowa City, USA

[4] Th. Bock, M. Maurer, F. van Meel, Th. Müller, Vehicle in the loop - Ein innovativer Ansatz zur Kopplung virtueller mit realer Erprobung, in ATZ 01/2008 Jahrgang 110.

[5] Michael Dick, Thomas Binder, Thomas Bock, Thomas Kriegel, Thomas Müller, Virtuelle Produktenstehung für Fahrzeug und Antrieb im KFZ, Vieweg Handbuch Kraftfahrzeugtechnik, ISBN-13: 978-3834802224

[6] Thomas Bock, Dissertation, Vehicle in the Loop - Test- und Simulationsumgebung für Fahrerassistenzsysteme, Cuvillier, ISBN: 978$3-86727-727-3$ 